\title{
SEPARABILITIES OF ARBITRARY ORDERS AND RELATED PROPERTIES ${ }^{1}$
}

\author{
R. G. LUBBEN
}

For the case of a metric space Gross ${ }^{1 a}$ established the equivalence of the following to each other and to other properties: (1) each point set is separable; (2) each uncountable point set contains a condensation point of itself. ${ }^{2}$ Sierpiński has shown that for a space $S$ Fréchet these two properties are not equivalent; also, he gives properties which are equivalent to (1) or to (2). ${ }^{3}$ These properties involve countable collections, and, thus, are concerned with the smallest transfinite cardinal. Appert ${ }^{4}$ generalizes by considering extensions to greater cardinals. He shows that certain of these properties imply others, but gives examples to show that they are not equivalent. ${ }^{5}$ In a recent paper the author showed the equivalence of the following properties for the case of a space $H$ Fréchet: (3) each uncountable point set contains a limit point of itself; (4) each uncountable point set either contains a condensation point of itself or is separable. ${ }^{6}$ This result supplements Sierpinski's work by showing that property (3), which for the case of metric spaces is equivalent to (1) and (2), is a common basis for them in that it necessarily involves elements of either the one or of the other. In Theorem 2 property (3) is shown to be a necessary and sufficient condition for properties which involve for each point set $M$ cardinals "as close as one pleases" to the power of $M$ in

${ }^{1}$ Presented to the Society, December 27, 1939, under the title Separabilities of higher orders and related properties.

1a Cf. W. Gross, Zur Theorie der Mengen in denen ein Distanzbegriff definiert ist, Sitzungsberichte der Kaiserlichen Akademie der Wissenschaften, part IIa, vol. 123 (1914), p. 801.

2 References to contributions by Fréchet may be found in his Espaces Abstraits et Leur Théorie Considérée Comme Introduction a l'Analyse Générale, Paris, 1928. The reader is referred to this treatise for definitions.

${ }^{3} \mathrm{~W}$. Sierpinski, Sur l'équivalence de trois propriétés des ensembles abstraits, Fundamenta Mathematicae, vol. 2 (1921), pp. 179-188. His results hold for more general spaces than the spaces $S$ he mentions.

${ }^{4}$ A. Appert, Propriêtés des Espaces Abstraits les Plus Généraux, Actualités Scientifiques et Industrielles, nos. 145 and 146, Paris, 1934, pp. 82-88. These extensions are considered in our Theorem 3.

${ }^{5}$ In Über höherstufige Separabilität und Kompaktheit, Japanese Journal of Mathematics, vol. 8 (1931), p. 114, Haratomi considers similar properties for the case of a metric space, for which they are equivalent; thus, his work is a generalization of Gross's.

${ }^{6}$ Concerning limiting sets in abstract spaces II, Transactions of this Society, vol. 43 (1938), p. 487. 
connection with such concepts as separability of higher orders, the density in $M$ of its limit points of higher orders, the extension of the Cantor-Bendixson theorem, and the "almost" perfect compactness of $M$. In Theorem 1 similar equivalences are established for orders which are not less than an arbitrary regular cardinal $\alpha$, provided that the following holds: $\left(3^{\prime}\right)$ each point set of power $\alpha$ contains a limit point of itself. Note that if $\alpha$ is the smallest transfinite cardinal, then $\left(3^{\prime}\right)$ is a corollary of $(3)$; and, if $\left(3^{\prime}\right)$ holds for a certain cardinal, it holds for greater cardinals. While our definitions differ from those of Appert, they yield a more systematic theory.

Definitions. If $\alpha$ is a cardinal, ${ }^{7}$ the point set $M$ is said to be strongly $\alpha$-separable provided that there exists $N$ such that (1) $\bar{N} \supset M \supset N$, and (2) either $N$ is countable or its power is less $\operatorname{than}^{8} \alpha$. If $\alpha$ is the power of $M$ and $M$ is strongly $\alpha$-separable, we say that $M$ is semi-separable. Thus, for a point set whose power $\alpha$ is aleph-one, the properties separability, semi-separability, and strong $\alpha$-separability are equivalent. If $M$ is a point set, $P$ is a point, and $\alpha$ is the smallest cardinal, $\beta$, such that there exists a neighborhood of $P, U$, for which the power of $U \cdot(M-P)$ is $\beta$, then $P$ is a limit point of $M$ of order $\alpha$. Thus, an isolated point of $M$ has order zero; a limit point has an order greater than zero; a condensation point has an order greater than aleph-zero; a complete limit point of $M$ has an order equal to the power of $M$. In a space $H$ Fréchet a limit point has an infinite order; at the end of this paper we give examples of spaces $V$ Fréchet in which there exist limit points of finite orders. A point of $M$ which has an order less than $\alpha$ is called an $\alpha$-isolated point of $M$. The point set $M$ is said to be almost perfectly $\alpha$-compact in itself provided that if $\alpha$ and $\delta$ are cardinals, $M \supset N, \alpha \leqq$ power of $N$, and $\delta<$ power of $N$, then there exists in $M$ a limit point of $N$ of an order at least as great as $\delta . M$ is almost perfectly compact in itself provided that if $M \supset N$ and $\delta$ is a transfinite cardinal which is less than the power of $N$, then $M$ contains a limit point of $N$ of an order at least as great as $\delta$; if, instead, we require that $\delta$ be the power of $N$, then $M$ is perfectly compact in itself. Thus, a countable set is almost perfectly compact in itself. Because of the equivalence of properties (6) and (1) of Theorem 2, if $M$ is almost perfectly compact in itself, the same property holds

${ }^{7}$ In this paper zero and the positive integers are included among the cardinals and the ordinals. Also, according to our usage, finite or vacuous sets are countable. For the properties of transfinite numbers see F. Hausdorff, Grundziige der Mengenlehre, Leipzig, 1914.

${ }^{8}$ If (2) is modified as follows, $M$ is $\alpha$-separable: Either $N$ is countable or its power is not greater than $\alpha$; cf. Appert, loc. cit., p. 83. 
for each of its subsets; an analogous proposition does not hold for perfectly compact sets. Consider the properties of $M:$ (a) it is perfectly compact in itself; it is almost perfectly $\alpha$-compact in itself, (b) for $\alpha=$ aleph-zero, or (c) for $\alpha=$ aleph-one; (d) it is almost perfectly compact in itself. Then (c) and (d) are equivalent; for, if $N$ is an uncountable subset of $M$ and $\delta<$ power of $N$, there exists a transfinite cardinal $\delta_{1}$ such that $\delta \leqq \delta_{1}<$ power of $N$; the conclusion follows. Each element of the sequence (a), (b), (c) implies any that follows it, but implies none that precede it. However, for a countable set in a space $H$ Fréchet (a) and (b) are equivalent. The following interpretation is suggestive: In (d) the power of $N$ is the "upper bound" of the orders of the limit points of $N$ which belong to $M$; in (a) this bound is attained.

THEOREM 1. Let $\alpha$ be a regular cardinal which is greater than alephzero, $T$ be a space $V$ Fréchet in which the operation of derivation of point sets is distributive, ${ }^{9}$ and the symbol $M$ denote an arbitrary point set in $T$. Then the following properties are equivalent.

(1) Each point set of power $\alpha$ contains a limit point of itself.

(2) Each point set either is strongly $\alpha$-separable, or it contains a limit point of itself which has an order not less than $\alpha$.

(3) If $M$ has a regular power $\beta$ and $\alpha \leqq \beta$, then either $M$ is semiseparable or $\beta$ is the power of each of the following subsets of $M$ : (a) that of all non- $\beta$-isolated points of $M$; (b) that of all complete limit points of $M$ which belong to $M$.

(4) Each point set is almost perfectly $\alpha$-compact in itself.

(5) Let $\alpha \leqq \beta$. (a) The power of the set of all isolated points of $M$ is less than $\beta$; (b) the power of the set of all $\beta$-isolated points of $M$ is not greater than $\beta$; (c) if $\beta<$ power of $M$, then $M$ and the set of its non- $\beta$ isolated points have the same power. ${ }^{10}$

Proof. Clearly, the properties (2) to (5) imply (1). Suppose that $E$ is a point set for which (2) does not hold. Then, for each point $P$ of $E$ there exists a subset $D(P)$ of $E$ such that $P$ is not an element of the closure of $E-D(P), P \varepsilon D(P)$, and the power of $D(P)$ is less than $\alpha$. Let $T$ be a well-ordered sequence of the elements of $E$ and $\delta$

${ }^{9}$ For example, $(E+F)^{\prime}=E^{\prime}+F^{\prime}$; this is equivalent to assuming that Hausdorff's Axioms A and B hold in T. Cf. Fréchet, loc. cit., pp. 172, 173, and 181; and Appert, loc. cit., pp. 23-27. A cardinal $\alpha$ is regular if it is not the sum of fewer than $\alpha$ cardinlas, each of which is distinct from zero and is less than $\alpha$; cf. W. Sierpinski, Hypothèse $d u$ Continu, Warsaw and Lwow, 1934, p. 152. With different hypotheses Appert obtains a result similar to part (5); loc. cit., p. 90.

${ }^{10} \beta$ is not necessarily regular. 
be the smallest ordinal in the number class $^{11} Z(\alpha)$. We shall define a well-ordered sequence $U=\left(q_{1}, q_{2}, \cdots, q_{\omega}, q_{\omega+1}, \cdots, q_{\beta}, \cdots\right)$ where $\beta<\delta$.

Proceed as follows: Let $q_{1}$ be the first element of $T$. Let $\beta$ be an ordinal which is greater than unity and is less than $\delta$; suppose that $q_{x}$ has been defined for all ordinals $x<\beta$, and let $U_{\beta}$ be the set of all such $q_{x}$ 's; let $S_{\beta}=\sum D\left(q_{x}\right)$, for $x<\beta$. Let $q_{\beta}$ be the first point of $T$ which does not belong to the closure of $S_{\beta}$.

We shall show that $q_{\beta}$ exists for all ordinals $\beta<\delta$. For, if there exist $\beta$ 's for which this is not true, there exists a smallest one, say $\lambda$. Then each point of $E$ belongs to the closure of $S_{\lambda}$. Then the power of $U_{\lambda}$ and the powers of each of the sets $D\left(q_{x}\right)$ for $x<\lambda$ are each less than $\alpha$. Since $\alpha$ is regular, the power of $S_{\lambda}$ is less than $\alpha$. Then, in contradiction to our supposition, $E$ is strongly $\alpha$-separable. Thus, $\lambda$ does not exist, and the power of $U$ is $\alpha$.

By definition $q_{\beta}$ is not a point or a limit point of $S_{\beta} \supset U_{\beta}$. Further, $S_{\beta+1}$, which contains $D\left(q_{\beta}\right)$, contains no point of $U-U_{\beta+1}$; thus $q_{\beta}$ is not a limit point of the latter. Since the operation of derivation is distributive and $U=q_{\beta}+U_{\beta}+\left(U-U_{\beta+1}\right)$, it follows that all points of $U$ are isolated in $U$. This contradicts (1); thus (1) implies (2).

Let $K$ be the set of all non- $\beta$-isolated points of $M$, and suppose that the power of $K$ is less than $\beta$. Then the power of $M-K=L$ is $\beta$. Since $L$ contains no limit point of itself of an order as great as $\beta$, it follows by (2) that there exists a subset $H$ of $L$ which is dense in $L$ and has a power less than $\beta$. Then $H+K$ is dense in $M$ and has a power less than $\beta$. Thus, $M$ is semi-separable, and (2) implies (3a); (3b) is (3a) for the case $\beta=$ power of $M$.

Suppose that (4) does not hold. Then there exist two point sets, $M$ and $N$, and two cardinals, $\beta_{1}$ and $\epsilon$, such that $\alpha \leqq \beta_{1}=$ power of $N$, $\epsilon<\beta_{1}, M \supset N$, and $N$ has no limit point in $M$ of an order as great as $\epsilon$. Let $\epsilon_{1}$ be the smallest cardinal which is greater than $\epsilon, \beta$ be the larger of $\alpha$ and $\epsilon_{1}$, and $\delta$ be the smallest ordinal in the number class $Z(\beta)$. Then $\beta$ is regular and does not exceed $\beta_{1}$. Let $F$ be a subset of $N$ having the power $\beta$; since $N \supset F, M$ contains no limit point of $F$ of an order as great as $\epsilon$. We shall define a well-ordered collection $G$ of subsets of $F$ such that the order type of $G$ is $\delta: G=\left(F_{1}, F_{2}, \cdots, F_{\omega}\right.$, $\left.\cdots, F_{m}, \cdots\right)$, where $m<\delta$.

Proceed as follows: Let $T$ be a well-ordered sequence of all subsets of $F$, and $F_{0}$ be the null set. Suppose that $m$ is an ordinal such that $m<\delta$ and $F_{x}$ has been defined for all ordinals $x$ which are less than $m$;

${ }^{11}$ Cf., F. Hausdorff, loc. cit., pp. 124-125. 
let $\Sigma_{m}=\sum F_{x}$, for $x<m$; let $F_{m}$ be the first element of $T$ which has a power less than $\beta$, which is a subset of $F-\Sigma_{m}$, and which is dense in $F-\Sigma_{m}$.

Suppose that the order type of $G$ is $\lambda$, where $\lambda<\delta$. Since $\beta$ is a regular cardinal, it follows that the power of $\Sigma_{\lambda}$ is less than $\beta$, and that of $F-\Sigma_{\lambda}$ is $\beta$. Since $F$ contains no limit point of itself of an order as great as $\epsilon$, it follows from (2) that $F-\Sigma_{\lambda}$ is strongly $\beta$-separable. Then $F_{\lambda}$ exists; and the supposition that $\lambda$ exists involves a contradiction.

Let $\lambda$ be the smallest ordinal in the number class $Z(\epsilon), P \varepsilon F_{\lambda}$, and $U$ be a neighborhood of $P$. Let $x$ and $y$ be ordinals such that $x<y \leqq \lambda$. Then $\Sigma_{y} \supset F_{x}$; since $F-\Sigma_{y} \supset F_{y}$, the product $F_{x} \cdot F_{y}$ is vacuous. Since $\Sigma_{y} \supset \Sigma_{x}, F-\Sigma_{x} \supset F-\Sigma_{y} \supset F_{y}$; then $F_{x}$ is dense on $F_{y}$. Thus for $x<\lambda$ the product $U \cdot F_{x}$ is nonvacuous. Hence, the power of $U \cdot \Sigma_{\lambda}$ is at least as great as $\epsilon$. Thus, we are involved in a contradiction, and (2) implies (4).

Property (1) implies (5a). Let $M_{b}$ be the set of all $\beta$-isolated points of $M$, and suppose that its power exceeds $\beta$. Let $\beta_{1}$ be the smallest cardinal which is greater than $\beta$. Then $\beta_{1}$ is regular, and by (4) each subset of $M_{b}$ having power $\beta_{1}$ contains a limit point of itself of order at least as great as $\beta$. Thus, we are involved in a contradiction; and (4) implies (5b), which implies (5c). Since (1) and (4) are equivalent, (4) implies (5).

THEOREM 2. In a space $V$ Frêchet in which the operation of derivation of point sets is distributive, the following are equivalent:

(1) Each uncountable set contains a limit point of itself.

(2) Each point set either contains a condensation point of itself, or it is separable.

(3) Each point set which has a regular power either is semi-separable, or it contains a complete limit point of itself.

(4) Each point set either is separable, or it has the same power as the set of all its condensation points which belong to it.

(5) Each point set which has a regular power either is semi-separable or it has the same power as the set of all its complete limit points which belong to it.

(6) Each point set is almost perfectly compact in itself.

(7) Part (5) of Theorem 1 holds for each transfinite cardinal $\beta$ such that aleph-zero $<\beta$.

Proof. This may be established by letting the $\alpha$ of Theorem 1 be aleph-one. In particular, to establish (4) apply the following parts of Theorem 1: (a) Part (3b), if aleph-one = power of $M$; (b) part (5c), if aleph-one < power of $M$. 
THEOREM 2A. The following modification of Theorem 1 is permissible: (I) Allow $\alpha$ to be any regular cardinal which is greater than unity; (II) replace the property " $M$ is strongly $\alpha$-separable" by the following: "there exists $N$ such that $\bar{N} \supset M \supset N$ and that the power of $N$ is less than $\alpha . "$

Note that the only finite cardinal which satisfies (I) is $\alpha=2$. Except for obvious modifications, the proof is like that of Theorem 1 . Note, however, the following: In considering the proof for (3) for the case power of $M=2$, let $P \varepsilon M \cdot M^{\prime}$; then $M-P$ is dense in $M$.

The following theorem gives relations between Appert's work and ours.

THEOREM 3. Let $\beta$ be transfinite, $\alpha$ be the cardinal which is next greatest to $\beta$, and $T$ be a space $V$ Fréchet. Edch of the following implies property (1) of Theorem $1 .^{12}$

(1) $T$ is $\beta$-perfectly separable.

(2) Each point set in $T$ is $\beta$-separable.

(3) Each point set in $T$ possesses the Lindeloff property of order $\beta$.

(4) Each point set of $T$ is $\beta$-condensed in itself. ${ }^{13}$

Comment. If $M$ is a point set in the space $T$ of Theorem 1 and $\alpha \leqq \lambda<$ power of $M$, then by (4) of that theorem $M$ contains a limit point of itself of an order at least as great as $\lambda$; that is, the upper bounds of the aggregates of the orders of the limit points which are involved in property (1) are the powers of the sets $M$ concerned. Further, if (1) holds for a given cardinal, it and the properties equivalent to it hold for all greater cardinals. If $\alpha$ is an arbitrary cardinal which is greater than unity, whether finite or transfinite, the following example establishes the existence of a space $T$ such that $\alpha$ is the smallest cardinal for which (1) holds relative to $T$.

Let $\alpha$ and $\beta$ be cardinals such that $\alpha<\beta$; let $S_{\alpha \beta}$ be a space whose points are the elements of an aggregate, of power $\beta$, and in which the set $U$ is a neighborhood of the point $P$ if and only if the following conditions hold: (1) $P \varepsilon U$; (2) the power of the complement of $U$ is

12 The converse is not true. Note that in Theorem 1 we have the additional hypothesis that the operation of derivation of point sets be distributive.

${ }^{13}$ Property (1) means that $T$ has a basis of a power that does not exceed $\beta$. Property (3) means that if a collection $G$ of sets covers the point set $M$, then there exists $H$ such that $G \supset H, H$ covers $M$, and the power of $H$ does not exceed $\beta$. The set $M$ is $\beta$-condensed in itself provided that if $\alpha$ is the smallest cardinal which is greater than $\beta$, $M \supset K$ and power of $K=\alpha$, then there exists a point $P$ of $M$ such that if $U$ is a neighborhood of $P$ then $\alpha$ is the power of the product of $K$ and the interior of $U$. Cf. Appert, loc. cit., pp. 82-88. 
less than $\alpha$, and is zero if $\alpha$ is finite. The following are properties of this space: (A) if $\alpha \geqq$ aleph-one, no point set of power $\alpha$ or greater is strongly $\alpha$-separable; (B) if $\alpha<\lambda$ and power $M=\lambda$, then $M$ is strongly $\lambda$-separable; (C) if $\alpha \leqq$ power $M$, then each point of $M$ is a complete limit point of $M$; thus property (1) of Theorem 1 holds; (D) if $\alpha$ is the smallest transfinite cardinal, the space is perfectly compact; (E) if $\alpha$ is transfinite, the space is a space $H$ Fréchet; $(F)$ the space satisfies the hypothesis of Theorem 1 , for $\alpha$ any cardinal. ${ }^{14}$ To establish (A) let power $M \geqq \alpha, M \supset N$, power $N<\alpha, P \varepsilon(M-N)$; then $P+\left(S_{\alpha \beta}-N\right)$ is a neighborhood of $P$, and $P$ is not a limit point of $N$. To establish (B) let $M \supset N, \alpha<$ power $M, \alpha \leqq$ power $N<$ power $M, P \varepsilon(M-N)$, and $U$ be a neighborhood of $P$; then power of $N \cdot\left(S_{\alpha \beta}-U\right)<\alpha$, and $N \cdot U$ is nonvacuous; thus $P$ is a limit point of $N$ and $N$ is dense in $M$. A consideration of the property "almost perfect compactness" suggests the question: Does there exist a space in which each infinite point set contains a complete limit point of itself? F. B. Jones gave an answer in the affirmative by suggesting the example in (D). If $\alpha$ is finite, each nonvacuous point set in $S_{\alpha \beta}$ contains a complete limit point of itself.

In Theorem 2, part (2), we consider two alternative properties, of which not more than one is required for each point set. For each of these properties Sierpiński ${ }^{15}$ gave an example of a space in which each point set has the given property, but not the alternative property. By taking the sum of his two spaces we have one in which the property that holds for a point set varies with the set. It would be interesting to give an example of a space for which (2) holds and for which each point set that satisfies one of the alternative conditions but not the other has a subset relative to which the converse is true.

The University of TeXas

${ }^{14}$ Cf. Appert, loc. cit., p. 25, and Fréchet, loc. cit., p. 186.

${ }^{15}$ Loc. cit., Fundamenta Mathematicae, vol. 2. 